\title{
Subjective Discomfort of TMS Predicts Reaction Times Differences in Published Studies
}

\author{
Nicholas Paul Holmes ${ }^{1 *}$ and Lotte Meteyard ${ }^{2}$ \\ ${ }^{1}$ School of Psychology, University of Nottingham, Nottingham, United Kingdom, ${ }^{2}$ School of Psychology and Clinical \\ Language Sciences, University of Reading, Reading, United Kingdom
}

Keywords: TMS, transcranial magnetic stimulation, side-effects, artifact, reaction times, posterior parietal cortex, inferior frontal gyrus, anterior temporal lobe

Transcranial magnetic stimulation (TMS) was developed 30 years ago, in part to decrease the peripheral side-effects associated with transcranial electrical stimulation (Barker, 1991). TMS has been effective in that aim, and great advances have been made over the past 30 years. TMS can still be uncomfortable and painful, however, as it stimulates excitable superficial tissue including scalp muscles and peripheral nerves (Maizey et al., 2013). This causes annoyance, pain, and muscle twitches (i.e., discomfort) that vary systematically across the scalp (Meteyard and Holmes, 2018). While superior and posterior scalp locations are associated with almost no discomfort, inferior frontal and temporal locations are associated with significant discomfort. This discomfort can include sharp pain and strong contractions of scalp, head, and neck muscles. In protocols where TMS and a behavioral task are separated by time ("off-line"), these peripheral side-effects of brain stimulation may not affect subsequent task performance. But, in protocols where TMS is applied simultaneously with the behavioral task ("on-line"), these side-effects of TMS might interfere significantly with performance.

Meteyard and Holmes (2018) found that participants' subjective ratings of the annoyance, pain, and muscle twitches caused by single pulse TMS was significantly and strongly correlated with changes in reaction time (RT) on two simple stimulus-response congruency tasks. Ratings of muscle twitches accounted for $43 \%$ of the variance in RT. TMS over parietal areas (e.g., P3/P4 electrode locations) resulted in a $9 \mathrm{~ms}$ decrease in RT. TMS over inferior locations (e.g., anterior temporal lobe) led to RT increases as large as $81 \mathrm{~ms}$. Thus, TMS-related peripheral side effects must be taken into account when studying the effects of on-line TMS. In particular, when effects of TMS are compared with a no TMS or sham condition, or when two TMS locations are compared, researchers need to control for differences in TMS-related discomfort.

For this Opinion, we investigated whether the TMS-related discomfort measured in our previous work could predict the reported differences in RT in studies published in the last 10 years. We searched for studies using on-line single-pulse TMS over the least uncomfortable (superior parietal) and most uncomfortable (anterior temporal, inferior frontal) brain areas. PubMed was searched with the query "(((TMS OR (transcranial magnetic stimulation)) AND parietal)) AND (“2008/01/01" [Date-Publication]:"3000" [Date-Publication]) AND (reaction OR response)." A second and third search replaced the term "parietal" with "anterior temporal" and "inferior frontal." Three hundred and seven results were returned for parietal, 14 for anterior temporal (1 more after extending the search to 20 years) and 65 for inferior frontal. Studies were included if we could access the full text, they were in English, studied healthy participants, and used on-line single-pulse TMS. We excluded studies using repetitive TMS, reporting only clinical data, or not containing RTs from a behavioral task. The parietal search focused on superior parietal or intraparietal locations; excluding more inferior supramarginal or angular gyri targets. Seventeen studies met the inclusion criteria (15 parietal, 2 inferior frontal). To increase the sample size, we relaxed the inclusion criteria 


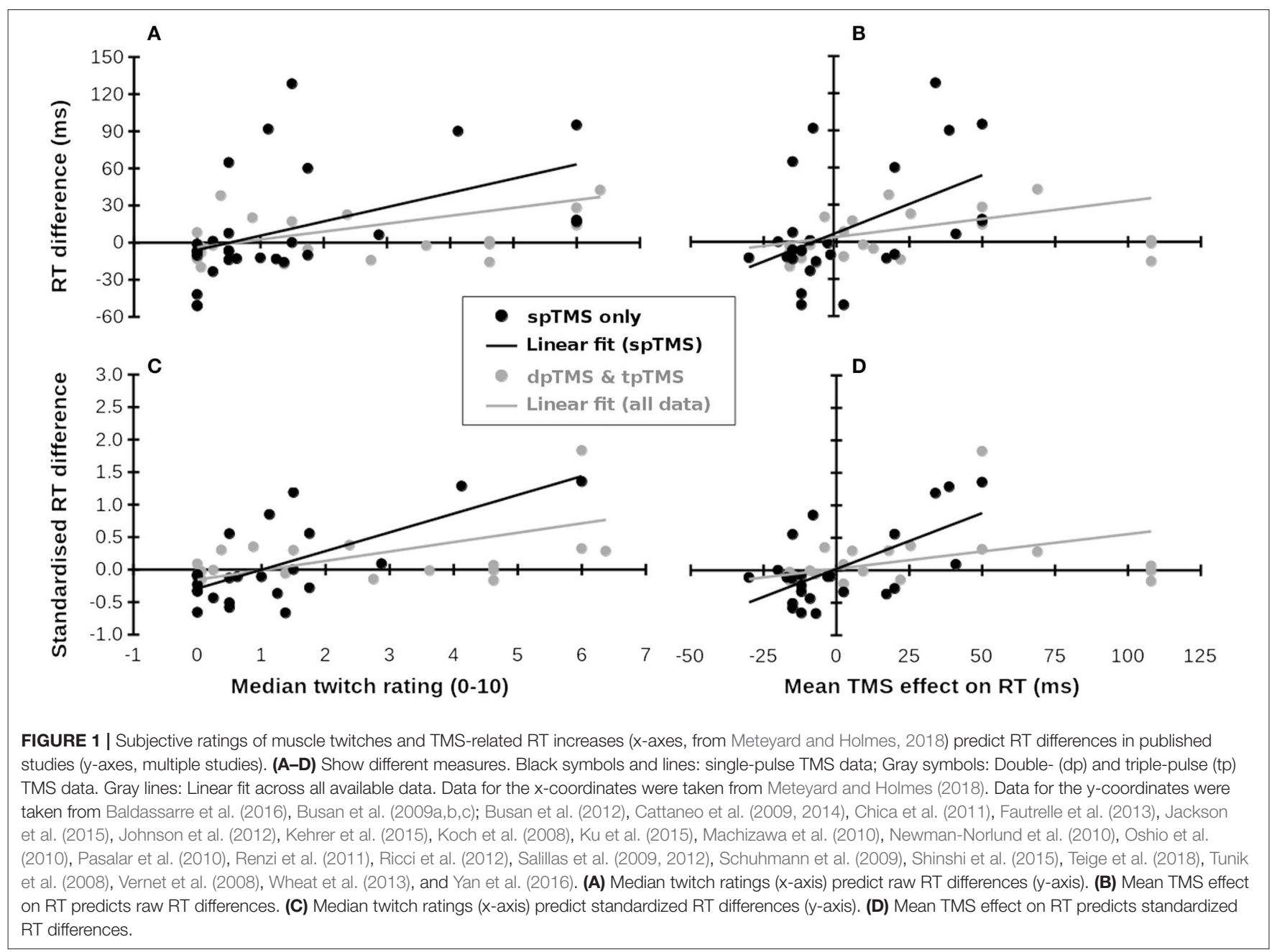

to include double-, and triple-pulse on-line TMS studies (total: 22 parietal, 6 inferior frontal, 2 anterior temporal).

From these articles, we extracted all the locations stimulated (including any additional non-parietal or non-frontal/temporal areas) and the RTs associated with those locations, averaging across other conditions. These reported locations were matched as closely as possible with those stimulated in Meteyard and Holmes (2018), which mostly corresponded to 10-10 EEG electrode locations. As predictor variables, we used the mean of median rating of muscle twitches and the mean effect of TMS on RT (i.e., change in RT with TMS as compared to no TMS), which were extracted from http://www.tms-smart.info. For the outcome variable, within each study, differences between RTs for each location and a control condition (no TMS, sham TMS, TMS over vertex, or the average of these) were calculated. Each study contributed at least one data point (mean $\pm S D=1.6 \pm 1.1$ data points per study, both for the 17 single-pulse studies and across all 30 studies). Where multiple TMS locations, groups, or experiments were reported, each study contributed multiple data points. RT differences and standardized RT differences (RT difference/pooled SD) were correlated with the per-location mean of median ratings of muscle twitches and effect of TMS on RT from Meteyard and Holmes (2018). 95\% confidence intervals for $r$-values were obtained by bootstrapping over 10,000 iterations. Results are plotted in Figure 1. Full data, analysis scripts, and supplementary figures can be found at https://osf.io/ f49vn/, in Supplementary Table 1, and at https://tms-smart.info.

For single-pulse TMS studies (28 samples), differences in RT between TMS and control conditions were significantly correlated with both the mean of median ratings of muscle twitches, $r_{26}=0.473,95 \% \mathrm{CI}=\{0.209,0.757\}, p=0.011$ (Figure 1A), and the mean effect of TMS on RT from Meteyard and Holmes (2018), $r_{26}=0.497\{0.173,0.740\}, p=0.007$ (Figure 1B). For each increment in twitch rating, RT increased by $11.5 \mathrm{~ms}$ ( $c f .15 \mathrm{~ms}$ in Meteyard and Holmes, 2018). This relationship strengthened when the observed RT data were standardized, both for twitches, $r_{24}=0.690\{0.265,0.872\}, p<$ 0.001 (Figure 1C), and RTs, $r_{24}=0.624\{0.165,0.846\}, p=0.001$ (Figure 1D). Each increment in twitch rating was associated with a change of $0.29 \mathrm{SD}$ in RT. Expanding the dataset to include double- and triple-pulse online TMS studies (Figure 1, gray symbols) weakened these relationships (twitches \& raw RT, $r_{47}$ 
$=0.365\{0.141,0.597\}, p=0.01$; RT \& raw RT, $r_{47}=0.272\{0.066$, $0.530\}, p=0.06$; twitches \& standardized RT, $r_{41}=0.529\{0.245$, $0.727\}, p<0.001$; RT \& standardized RT, $r_{41}=0.346\{0.138$, $0.613\}, p=0.023)$. TMS over superior parietal cortex, and over other scalp regions where little discomfort is felt, results in a small decrease in RT (bottom-left of Figures 1A-D), whereas TMS at scalp locations causing significant discomfort generally increases RT (top-right of Figures 1A-D). In all analyses, the bootstrapped 95\% confidence intervals for the correlation coefficients did not include 0 . In an additional check for robustness of the correlations, each dataset was submitted to 10,000 iterations of a "leave $\mathrm{N}$ out" analysis. On each iteration, the original dataset was re-analyzed, each time leaving out a randomly-selected $\mathrm{N}$ datapoints, where $\mathrm{N}$ varied between 1 and 20. The 95\% confidence interval for all $8 r$-values reported above included $r=$ 0 only when at least 13 datapoints had been left out of the analysis. These analyses suggest that the observed correlation is positive and does not depend on particular datapoints being included.

Subjective ratings of muscle twitch strength can predict the effects of TMS on RT at a range of scalp locations, across a range of tasks, and across a range of TMS protocols. Two qualifications should be made. First, the reported correlations may be a slight underestimation of the true effect. Across studies, the TMS intensity used (maximum stimulator output) was inverselyrelated both to predicted discomfort $\left[r_{(28)}=-0.507, p=\right.$ 0.004 ], and to predicted RT changes associated with stimulation at that site [from Meteyard and Holmes, 2018 data, $r_{(28)}=$ $-0.524, p=0.003$ ], although this was not significant for the RT differences reported by the studies themselves $\left[r_{(26)}=-0.124, p\right.$ $=0.55]$. This could be due to the finding that higher discomfort is associated with lower scalp-to-cortex distances (Meteyard and Holmes, 2018), thus requiring lower TMS intensity. Or, it could be that researchers use lower TMS intensities when their participants report greater discomfort. Second, the reported

\section{REFERENCES}

Baldassarre, A., Capotosto, P., Committeri, G., and Corbetta, M. (2016). Magnetic stimulation of visual cortex impairs perceptual learning. NeuroImage 143, 250-255. doi: 10.1016/j.neuroimage.2016.08.063

Barker, A. T. (1991). An introduction to the basic principles of magnetic nerve stimulation. J. Clin. Neurophysiol. 8, 26-37.

Busan, P., Barbera, C., Semenic, M., Monti, F., Pizzolato, G., Pelamatti, G., et al. (2009a). Effect of transcranial magnetic stimulation (TMS) on parietal and premotor cortex during planning of reaching movements. PLoS ONE 4:e4621. doi: 10.1371/journal.pone.0004621

Busan, P., Jarmolowska, J., Semenic, M., Monti, F., Pelamatti, G., Pizzolato, G., et al. (2009b). Involvement of ipsilateral parieto-occipital cortex in the planning of reaching movements: evidence by TMS. Neurosci. Lett. 460, 112-116. doi: 10.1016/j.neulet.2009.05.028

Busan, P., Monti, F., Semenic, M., Pizzolato, G., and Battaglini, P. P. (2009c). Parieto-occipital cortex and planning of reaching movements: a transcranial magnetic stimulation study. Behav. Brain Res. 201, 112-119. doi: 10.1016/j.bbr.2009.01.040

Busan, P., Zanon, M., Vinciati, F., Monti, F., Pizzolato, G., and Battaglini, P. P. (2012). Transcranial magnetic stimulation and preparation of visuallyguided reaching movements. Front. Neuroeng. 5:18. doi: 10.3389/fneng.2012. 00018 correlations were weaker when the double- and triple-pulse TMS studies' data were included. This could be due to genuine differences in discomfort between single-pulse and multi-pulse TMS, or to the greater heterogeneity of study types and TMS parameters when all TMS protocols were included.

We cannot conclude that the results of the reviewed studies merely reflect differences in the peripheral side-effects of TMS, since we deliberately averaged across experimental conditions (but not scalp locations) within each study. Instead, we can conclude that RTs under TMS differ systematically across scalp locations (i.e., not necessarily caused by differences in, or the effects of TMS on, the underlying brain regions). Direct comparisons of RT between TMS at different scalp locations are confounded by differences in these peripheral side-effects, and must be interpreted with caution.

The data from Meteyard and Holmes (2018) has been used to create interactive maps available at http://www.tmssmart.info. Researchers can use these interactive maps to select control sites that may help account for TMS-related discomfort. In the next 30 years of studies using TMS, we recommend systematically controlling for the side-effects associated with magnetic stimulation of the scalp, for example by choosing control sites with similar levels of discomfort.

\section{AUTHOR CONTRIBUTIONS}

All authors listed have made a substantial, direct and intellectual contribution to the work, and approved it for publication.

\section{SUPPLEMENTARY MATERIAL}

The Supplementary Material for this article can be found online at: https://www.frontiersin.org/articles/10.3389/fpsyg. 2018.01989/full\#supplementary-material

Cattaneo, Z., Lega, C., Gardelli, C., Merabet, L. B., Cela-Conde, C. J., and Nadal, M. (2014). The role of prefrontal and parietal cortices in esthetic appreciation of representational and abstract art: a TMS study. NeuroImage 99, 443-450. doi: 10.1016/j.neuroimage.2014. 05.037

Cattaneo, Z., Rota, F., Walsh, V. Z., Vecchi, T., and Silvanto, J. (2009). TMS-adaptation reveals abstract letter selectivity in the left posterior parietal cortex. Cereb. Cortex 19, 2321-2325. doi: 10.1093/cercor/b hn249

Chica, A. B., Bartolomeo, P., and Valero-Cabré, A. (2011). Dorsal and ventral parietal contributions to spatial orienting in the human brain. J. Neurosci. 31, 8143-8149. doi: 10.1523/JNEUROSCI.5463-10.2010

Fautrelle, L., Gueugnon, M., Barbieri, G., and Bonnetblanc, F. (2013). Interhemispheric remapping between arm proprioception and vision of the hand is disrupted by single pulse TMS on the left parietal cortex. Brain Cogn. 82, 146-151. doi: 10.1016/j.bandc.2013.03.002

Jackson, R. L., Lambon-Ralph, M. A., and Pobric, G. (2015). The timing of anterior temporal lobe involvement in semantic processing. J. Cogn. Neurosci. 27, 1388-1396. doi: 10.1162/jocn_a_00788

Johnson, J. S., Kundu, B., Casali, A. G., and Postle, B. R. (2012). Task-dependent changes in cortical excitability and effective connectivity: a combined TMS-EEG study. J. Neurophysiol. 107, 2383-2392. doi: 10.1152/jn.00707. 2011 
Kehrer, S., Kraft, A., Koch, S. P., Kathmann, N., Irlbacher, K., and Brandt, S. A. (2015). Timing of spatial priming within the frontoparietal attention network: a TMS study. Neuropsychologia 74, 30-36. doi: 10.1016/j.neuropsychologia.2014.11.017

Koch, G., Fernandez Del Olmo, M., Cheeran, B. J., Schippling, S., Caltagirone, C., Driver, J., et al. (2008). Functional interplay between posterior parietal and ipsilateral motor cortex revealed by twin-coil transcranial magnetic stimulation during reach planning toward contralateral space. J. Neurosci. 28, 5944-5953. doi: 10.1523/JNEUROSCI.0957-08.2008

Ku, Y., Zhao, D., Hao, N., Hu, Y., Bodner, M., and Zhou, Y. (2015). Sequential roles of primary somatosensory cortex and posterior parietal cortex in tactilevisual cross-modal working memory: a single-pulse transcranial magnetic stimulation (spTMS) study. Brain Stim. 8, 88-91. doi: 10.1016/j.brs.2014. 08.009

Machizawa, M. G., Kalla, R., Walsh, V., and Otten, L. J. (2010). The time course of ventrolateral prefrontal cortex involvement in memory formation. $J$. Neurophysiol. 103, 1569-1579. doi: 10.1152/jn.90937.2008

Maizey, L., Allen, C. P., Dervinis, M., Verbruggen, F., Varnava, A., Kozlov, M., et al. (2013). Comparative incidence rates of mild adverse effects to transcranial magnetic stimulation. Clin. Neurophysiol. 124, 536-544. doi: 10.1016/j.clinph.2012.07.024

Meteyard, L., and Holmes, N. P. (2018). TMS SMART - scalp mapping of annoyance ratings and twitches caused by transcranial magnetic stimulation. J. Neurosci. Meth. 299, 34-44. doi: 10.1016/j.jneumeth.2018.02.008

Newman-Norlund, R. D., Ondobaka, S., van Schie, H. T., van Elswijk, G., and Bekkering, H. (2010). Virtual lesions of the ifg abolish response facilitation for biological and non-biological cues. Front. Behav. Neurosci. 4:5. doi: 10.3389 /neuro.08.005.2010

Oshio, R., Tanaka, S., Sadato, N., Sokabe, M., Hanakawa, T., and Honda, M. (2010). Differential effect of double-pulse TMS applied to dorsal premotor cortex and precuneus during internal operation of visuospatial information. NeuroImage 49, 1108-1115. doi: 10.1016/j.neuroimage.2009.07.034

Pasalar, S., Ro, T., and Beauchamp, M. S. (2010). TMS of posterior parietal cortex disrupts visual tactile multisensory integration. Eur. J. Neurosci. 31, 1783-1790. doi: 10.1111/j.1460-9568.2010.07193.x

Renzi, C., Vecchi, T., Silvanto, J., and Cattaneo, Z. (2011). Overlapping representations of numerical magnitude and motion direction in the posterior parietal cortex: a TMS-adaptation study. Neurosci. Lett. 490, 145-149. doi: 10.1016/j.neulet.2010.12.045

Ricci, R., Salatino, A., Li, X., Funk, A. P., Logan, S. L., Mu, Q., et al. (2012). Imaging the neural mechanisms of TMS neglect-like bias in healthy volunteers with the interleaved TMS/FMRI technique: preliminary evidence. Front. Hum. Neurosci. 6:326. doi: $10.3389 /$ fnhum.2012.00326

Salillas, E., Basso, D., Baldi, M., Semenza, C., and Vecchi, T. (2009). Motion on numbers: transcranial magnetic stimulation on the ventral intraparietal sulcus alters both numerical and motion processes. J. Cogn. Neurosci. 21, 2129-2138. doi: 10.1162/jocn.2008.21157

Salillas, E., Semenza, C., Basso, D., Vecchi, T., and Siegal, M. (2012). Single pulse TMS induced disruption to right and left parietal cortex on addition and multiplication. NeuroImage 59, 3159-3165. doi: 10.1016/j.neuroimage.2011.10.093

Schuhmann, T., Schiller, N. O., Goebel, R., and Sack, A. T. (2009). The temporal characteristics of functional activation in broca's area during overt picture naming. Cortex 45, 1111-1116. doi: 10.1016/j.cortex.2008.10.013

Shinshi, M., Yanagisawa, T., Hirata, M., Goto, T., Sugata, H., Araki, T., et al. (2015). Temporospatial identification of language-related cortical function by a combination of transcranial magnetic stimulation and magnetoencephalography. Brain Behav. 5:317. doi: 10.1002/brb3.317

Teige, C., Mollo, G., Millman, R., Savill, N., Smallwood, J., Cornelissen, P. L., et al. (2018). Dynamic semantic cognition: characterising coherent and controlled conceptual retrieval through time using magnetoencephalography and chronometric transcranial magnetic stimulation. Cortex 103, 329-349. doi: 10.1016/j.cortex.2018.03.024

Tunik, E., Lo, O. Y., and Adamovich, S. V. (2008). Transcranial magnetic stimulation to the frontal operculum and supramarginal gyrus disrupts planning of outcome-based hand-object interactions. J. Neurosci. 28, 14422-14427. doi: 10.1523/JNEUROSCI.4734-08.2008

Vernet, M., Yang, Q., Daunys, G., Orssaud, C., and Kapoula, Z. (2008). TMS of the posterior parietal cortex delays the latency of unpredictable saccades but not when they are combined with predictable divergence. Brain Res. Bull. 76, 50-56. doi: 10.1016/j.brainresbull.2007.11.007

Wheat, K. L., Cornelissen, P. L., Sack, A. T., Schuhmann, T., Goebel, R., and Blomert, L. (2013). Charting the functional relevance of broca's area for visual word recognition and picture naming in dutch using fmri-guided TMS. Brain Lang. 125, 223-230. doi: 10.1016/j.bandl.2012. 04.016

Yan, Y., Wei, R., Zhang, Q., Jin, Z., and Li, L. (2016). Differential roles of the dorsal prefrontal and posterior parietal cortices in visual search: a TMS study. Sci. Rep. 6:30300. doi: 10.1038/srep30300

Conflict of Interest Statement: The authors declare that the research was conducted in the absence of any commercial or financial relationships that could be construed as a potential conflict of interest.

Copyright $\odot 2018$ Holmes and Meteyard. This is an open-access article distributed under the terms of the Creative Commons Attribution License (CC BY). The use, distribution or reproduction in other forums is permitted, provided the original author(s) and the copyright owner(s) are credited and that the original publication in this journal is cited, in accordance with accepted academic practice. No use, distribution or reproduction is permitted which does not comply with these terms. 